Check for updates

Cite this: RSC Adv., 2018, 8, 27935

Received 16th June 2018

Accepted 7th July 2018

DOI: $10.1039 / c 8 r a 05165 h$

rsc.li/rsc-advances

\section{Development of a high-throughput and sensitive assay of fusion genes in lung cancer by array-based MALDI-TOFMS $\uparrow$}

\author{
Han-Tao Wu, ${ }^{a}$ Kun Li, ${ }^{a}$ Gang Wang, ${ }^{a}$ Xue-Xi Yang, ${ }^{a}$ Anna Zhu, ${ }^{b}$ Xu-Ping Xu, ${ }^{\text {b }}$ Ming Li, ${ }^{a}$ \\ Ying-Song $\mathrm{Wu}^{* a}$ and Tian-Cai Liu (iD *a
}

\begin{abstract}
ALK (anaplastic lymphoma kinase gene), ROS1 (ros proto-oncogene 1) and RET (ret proto-oncogene) fusions are oncogenic drivers in non-small cell lung cancer (NSCLC). Methods like fluorescence in situ hybridization (FISH) and immunohistochemistry (IHC) are highly sensitive but subjectively analyzed, labor intensive, expensive and unsuitable for multiple fusion gene screening. This study aimed to establish a highthroughput, sensitive and cost-effective screening method (array-based matrix-assisted laser desorption/ ionization time-of-flight mass spectrometry, array-based MALDI-TOFMS) for ALK, ROS1 and RET fusion detection. This method was established with three fusion gene positive cell lines (H2228, ALK positive; HCC78, ROS1 positive; LC-2/AD, RET positive) and negative samples. Then, 34 clinical samples were selected and detected by Sanger sequencing, next generation sequencing (NGS) and array-based MALDITOFMS. The results were compared and analyzed and Sanger sequencing was considered the standard. 7 cases showed ALK fusions, 1 case showed ROS1 fusions, no case showed RET fusions and 4 cases were both ALK and ROS1 fusions. Results showed that array-based MALDI-TOFMS was 100\% concordant with Sanger sequencing and NGS $82.3 \%$. In this study, we reported the utility of array-based MALDI-TOFMS in the assessment of ALK, ROS1 and RET fusions in routine lung biopsies of FFPE and fresh tissue specimens. Besides, this method may also be applied to the diagnosis, monitoring and prognosis of illness.
\end{abstract}

\section{Introduction}

Lung cancer is the leading cause of cancer-related mortality worldwide, ${ }^{\mathbf{1}, \mathbf{2}}$ with non-small cell lung cancer accounting for $85 \%$ of all lung cancer cases, and its prognosis remains very poor., ${ }^{3,4}$

The anaplastic lymphoma kinase gene $(A L K)$ encodes a receptor tyrosine kinase that is aberrant in a variety of malignancies. Approximately 3-7\% of NSCLC patients harbor $A L K$ fusions and they are more commonly found in light smokers (<10 pack years) and/or never-smokers. ${ }^{5,6}$ DNA rearrangement leads to expression of a constitutively active protein kinase. Tumors harboring $A L K$ rearrangements rely specifically on the chimeric oncoprotein for progression and are sensitive to ALK inhibitors, such as crizotinib, a drug approved by US Food and Drug Administration for the treatment of $A L K$ fusionpositive non-small cell lung cancer (NSCLC). ${ }^{5,7}$

${ }^{a}$ Institute of Antibody Engineering, School of Laboratory Medicine and Biotechnology, Southern Medical University, Guangzhou 510515, Guangdong, P. R. China. E-mail: wg@smu.edu.cn; liutc@smu.edu.cn; Fax: +86-20-37247604; Tel: +86-20-62789355 ${ }^{b}$ Guangzhou Darui Biotechnology Co. Ltd., Guangzhou 510665, China

$\dagger$ Electronic supplementary information (ESI) available. See DOI: $10.1039 / \mathrm{c} 8 \mathrm{ra} 05165 \mathrm{~h}$
The ROS proto-oncogene 1, receptor tyrosine kinase (ROS1) is a member of the insulin receptor family. Approximately $2 \%$ of lung tumors harbor ROS1 fusions and, like $A L K$ fusions, ROS1 fusions are more commonly found in light smokers $(<10$ pack years) and/or never-smokers. ${ }^{8}$ In an expansion cohort of a phase I study, 50 patients with ROS1-positive NSCLC demonstrated a $72 \%$ response rate and $\mathbf{1 9 . 2}$ month median progression-free survival interval when treated with crizotinib. ${ }^{9}$

The ret proto-oncogene (RET) gene, located on chromosome 10 , encodes a receptor tyrosine kinase belonging to the RET family. Several recent cancer genome sequencing studies identified RET fusions in 1-2\% of NSCLC, most frequently involving kinesin family member $5 \mathrm{~B}$ (KIF5B) and coiled-coil domain containing 6 (CCDC6) as fusion partners. ${ }^{10}$ In addition, cells with $R E T$ fusions may be sensitive to multitargeted kinase inhibitors such as sunitinib, sorafenib and vandetanib. ${ }^{\mathbf{1 1}}$

Currently, the gold standard method for gene fusion detection in lung cancer is fluorescence in situ hybridization (FISH) ${ }^{\mathbf{1 2}}$ However, this technique is expensive, labor intensive and requires assessment by a pathologist meaning that the results are subjective. ${ }^{13}$ Other methods like immunohistochemistry (IHC) and reverse transcription polymerase chain reaction (RTPCR) also have their disadvantages, like the false positive rate of RT-PCR ${ }^{\mathbf{1 4}}$ and the low and partial expression of ALK oncoproteins in IHC. ${ }^{15}$ 
NanoString technology is a promising new method for detecting fusions. Adriane and co-workers reported the usefulness of NanoString in the assessment of $A L K$ fusions in 43 lung biopsies of FFPE specimens, which were validated previously by FISH/IHC. ${ }^{16}$ However, a larger number of samples still need to be assessed for this to be validated.

Consequently, a high-throughput, sensitive and cost-effective method that detects and evaluates $A L K$, ROS1 and RET fusion gene is urgently needed. MALDI-TOF was widely applied in the detection of protein and nucleic acid. Based on the conservation protein peak, this method can distinguish different species of microorganism. ${ }^{\mathbf{1 7}}$ David and his co-workers made a proof of principle of their MALDI-TOFMS approach in the clinical setting using recently isolated Fusarium strains demonstrated its validity. ${ }^{18}$ Joyner utilized MALDI-TOFMS to monitor the kinetics and products of RNA cleavage and proved the method is a rapid, accurate, highly-detailed and semi-quantitative analysis of RNA cleavage. ${ }^{19}$ In this study, we combined a transcriptomic platform to handle RNA extracted from samples and array to perform highthroughput. Then we validated this method with three fusion gene positive cell lines and negative samples and tested this method with 34 formalin-fixed paraffin-embedded (FFPE) and fresh tissue samples from Chinese population and compared the results with Sanger sequencing and NGS.

\section{Experimental}

\subsection{Reagents and instruments}

The following materials were used in this study: lung cancer ALK/ROS1/RET gene Fusion Detection Kit (Guangzhou Darui Biotechnology Co. Ltd, Guangzhou, China), Recover All тм Total Nucleic Acid Isolation Kit (Thermo, MA, USA), Qiagen RNeasy Mini kit (Qiagen, Valencia, Germany), Invitrogen SuperScript ${ }^{\circledR}$ VILO cDNA synthesis kit (Thermo, MA, USA), $\mathrm{H}_{2} \mathrm{O}$ (SigmaAldrich, USA), Qubit 3.0 ${ }^{\mathrm{TM}}$ fluorimeter (Thermo, MA, USA). Veriti Dx Thermal 96 well Cycler (Life Technologies, New York, USA), time of flight mass spectrometry detection system (DR MassARRAY) (Guangzhou Darui Biotechnology Co. Ltd, Guangzhou, China). All processes were followed by the manufacturer recommendations (Fig. 1).

\subsection{Preparation of samples}

2.2.1 Samples. Three cell lines obtained from Cobioer Biotechnology Company (H2228, ALK positive ${ }^{20}$; HCC78, ROS1 positive ${ }^{21}$; LC-2/AD, RET positive ${ }^{21}$ ) cultured in $37{ }^{\circ} \mathrm{C}, 5 \% \mathrm{CO}_{2}$ for two days and negative samples that had been previously tested by Sanger sequencing. A total of 34 surgically resected NSCLC samples which were not randomly collected but partly validated by FISH previously and 128 negative samples form DaAn Gene Co. Ltd. of Sun Yat-sen University were collected and maintained in $-20{ }^{\circ} \mathrm{C}$.

2.2.2 Ethical approval. Informed consent was obtained from each patient and the study was conducted in accordance with the Declaration of Helsinki and was approved by the Local Ethics Committee of Southern Medical University.

\subsection{Establishment of array-based MALDI-TOFMS}

2.3.1 RNA extraction. For FFPE samples, 2 to 10 sections (7$\mu \mathrm{m}$ thickness) were used to isolate RNA and $30 \mathrm{mg}$ for fresh tissue samples, and all of the samples used Recover All ${ }^{\mathrm{TM}}$ Total Nucleic Acid Isolation Kit. For the cell lines total RNA was isolated using the Qiagen RNeasy Mini kit. RNA concentration was assessed with Qubit.

2.3.2 Conversion of cDNA. Total RNA was converted to cDNA using an Invitrogen SuperScript ${ }^{\circledR}$ VILO cDNA synthesis kit with random hexamer priming according to manufacturer's recommendations and RNA was maintained in $-80{ }^{\circ} \mathrm{C}$.

2.3.3 Array-based MALDI-TOFMS. Multiplex screening of the samples was performed using the array-based MALDITOFMS LungFusion panel. The 16 extension primers were designed as listed in Table 1. Each extension primer was companied with 250 copies competitive fragments, and they were designed to evaluate the amount of fusion gene expression. By comparing the expression quantity of $3^{\prime}$ and $5^{\prime}$ section of fusion genes, the status was determined. The primers designed for $E M L 4$ were to ensure the samples obtained from lung tissues and the primers designed for $G A P D H$ were to evaluate the quantity of gDNA.

This method contained 5 steps, PCR, Shrimp alkaline phosphatase reaction (SAP), extension reaction, preparation of array and analysis. The thermocycling cocktail and condition are listed at Tables S1-S3. $\dagger$

Array was maintained at room temperature. After liquid handling, $40 \mu \mathrm{L}$ resin was added into extension product to adsorb saline ions, then it was sealed and spotted six dots a time on the array by dispenser. And $15 \mathrm{~nL}$ extension product was spotted on the array. While finishing spotting, array was sent into MALDI-TOFMS. The intensity of laser is 500 and the whole process was maintained at $4{ }^{\circ} \mathrm{C}$.

The results were analyzed by Type 4.0 , the report was given by comparing the unbalanced expression of $3^{\prime}$ and $5^{\prime}$ of genes.

2.3.4 Sanger sequencing. For the fusion gene variants of $A L K$, ROS1 and RET fusions, PCR primers were designed using Primer Premier 5.0 (http://www.bio-soft.net). PCR was performed by Veriti Dx Thermal 96 well Cycler. cDNA was converted from RNA, then cDNA was quantified using Qubit 3.0. The thermocycling cocktail and conditions were as follows. The sequencing process was followed to Sanger Sequencing Protocol and thermocycling cocktail and conditions are listed at Tables S4 and S5. $\dagger$ After that, we analyzed the results with Chromas V1.62 (http://www.sangon.com).

2.3.5 Next-generation sequencing. We used the ThermoFisher NGS Fusion Assay for fusion gene sequencing including AmpliSeq assay technology and Ion Torrent sequencing for the targeted sequencing of known and de novo fusion junctions for $A L K$, ROS1 and RET. The concentration of RNA was evaluated by Qubit followed by reverse transcription by multiplex PCR using a single primer pool. Briefly, libraries were prepared and barcoded sequencing adapters ligated to the resulting amplicons. Libraries were pooled, amplified in emulsion PCR and sequenced on the Ion Torrent PGM instrument. Samples were batched on a single 318 PGM chip to give $>150000$ reads per sample. 


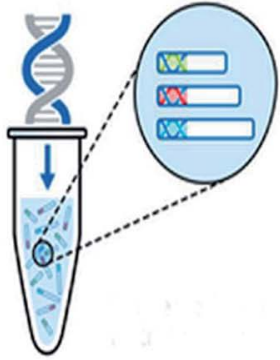

PCR, SAP and extension $P C R$, SAP and extension reaction were all processed in one single well.

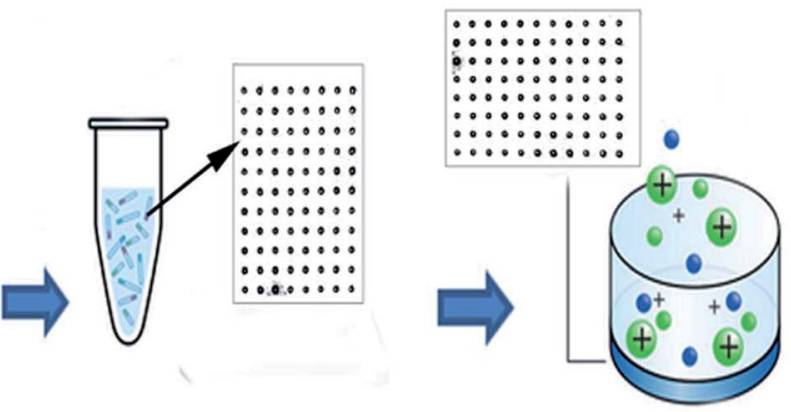

Preparation of array. Transfer a small amount of sample to a single pad on the array.

\section{MALDI}

Extension products were ionized by laser. Multiple tests can be run in a single array.

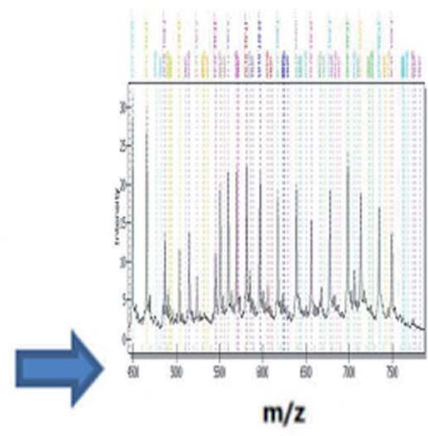

TOF Mass Spectra The mass of extension product was detected by TOF Mass Spectra.

ALK - anaplastic lymphoma receptor tyrosine kinase

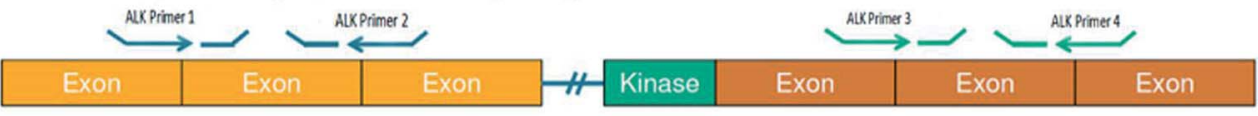

RET - ret proto-oncogene
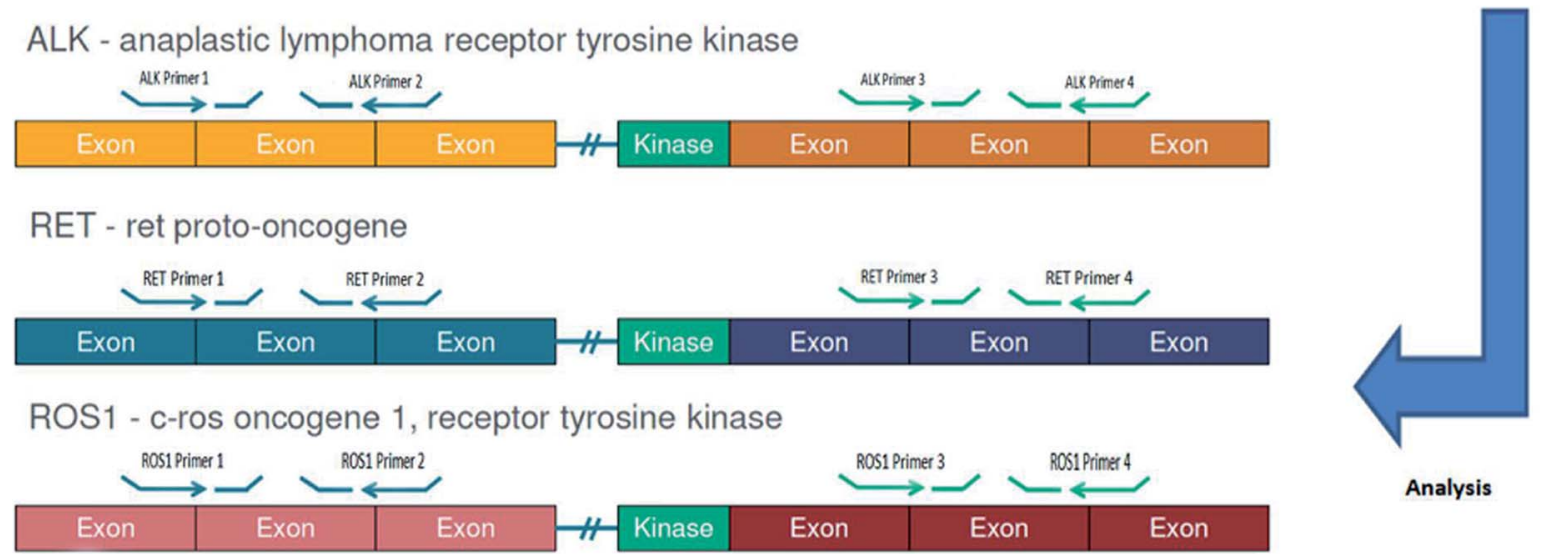

Reports were given by detecting the unbalanced expression of $3^{\prime}$ and $5^{\prime}$ of genes.

Fig. 1 Workflow of array-based MALDI-TOFMS. RNA was extracted from samples and converted to cDNA, then PCR, SAP and extension were performed, extension primers were extended one base and ionized by laser. The mass of extended primers was detected by MALDI-TOFMS. By detecting and comparing the expression of $3^{\prime}$ and $5^{\prime}$ of fusion gene, a $Z$ score would be given to evaluate fusion.

Torrent Suite software server for alignment was used to transfer sample data and Ion Reporter software v4.4 for variant calling. After sequencing, reads are aligned and for samples with more than 34000 mapped reads, fusions were detected when the sequence of one gene was found relocated to that of

Table 1 Number and position of extension primers ${ }^{a}$

\begin{tabular}{lllllllll}
\hline Primer position & \multicolumn{1}{l}{ ALK } & \multicolumn{3}{l}{ ROS1 } & \multicolumn{2}{l}{ RET } & EML4 & GAPDH \\
& $3^{\prime}$ & $5^{\prime}$ & $3^{\prime}$ & $5^{\prime}$ & $3^{\prime}$ & $5^{\prime}$ & & \\
Primer number & 2 & 3 & 2 & 2 & 2 & 2 & 2 & 2
\end{tabular}

${ }^{a}$ Primers for $3^{\prime}$ and $5^{\prime}$ fusion genes were to ensure fusion types. Primers for EML4 were to ensure the samples resource and the primers designed for $G A P D H$ were to evaluate gDNA quantity. another gene at the breakpoint. A fusion was called when more than 40 fusion reads supported the breakpoint. Fusions were also inferred qualitatively from differential expression data, where expression of the $5^{\prime}$ and $3^{\prime}$ ends of a transcript were significantly imbalanced in fusion samples compared with normal samples.

Table 2 Median and variance of $A L K, R O S 1$ and RET

\begin{tabular}{llllll}
\hline ALK median & $\begin{array}{l}A L K \\
\text { variance }\end{array}$ & $\begin{array}{l}\text { ROS1 } \\
\text { median }\end{array}$ & $\begin{array}{l}\text { ROS1 } \\
\text { variance }\end{array}$ & $\begin{array}{l}\text { RET } \\
\text { median }\end{array}$ & $\begin{array}{l}\text { RET } \\
\text { variance }\end{array}$ \\
\hline 0.519 & 0.126 & 0.504 & 0.092 & 0.518 & 0.105
\end{tabular}


Table 3 Summary of fusion detection results in cell lines. $\mathrm{P}=$ positive, $\mathrm{N}=$ negative

\begin{tabular}{|c|c|c|c|c|c|c|c|c|c|}
\hline & \multicolumn{3}{|c|}{$\begin{array}{l}\text { Array-based } \\
\text { MALDI-TOF }\end{array}$} & \multicolumn{3}{|l|}{ NGS } & \multicolumn{3}{|c|}{ Sanger sequencing } \\
\hline & ALK & ROS1 & RET & ALK & ROS1 & RET & ALK & ROS1 & ALK \\
\hline H2228 & $\mathrm{P}$ & $\mathrm{N}$ & $\mathrm{N}$ & $\begin{array}{l}\text { EML4-ALK } \\
(\text { E13, A20) }\end{array}$ & $\mathrm{N}$ & $\mathrm{N}$ & $\begin{array}{l}\text { EML4-ALK } \\
\text { (E13, A20) }\end{array}$ & $\mathrm{N}$ & $\mathrm{N}$ \\
\hline HCC78 & $\mathrm{N}$ & $\mathrm{P}$ & $\mathrm{N}$ & $\mathrm{N}$ & $\begin{array}{l}\text { SLC34A2-ROS1 } \\
\text { (S4, R34) }\end{array}$ & $\mathrm{N}$ & $\mathrm{N}$ & $\begin{array}{l}\text { SLC34A2-ROS1 } \\
\text { (S4, R34) }\end{array}$ & $\mathrm{N}$ \\
\hline Negative samples & $\mathrm{N}$ & $\mathrm{N}$ & $\mathrm{N}$ & $\mathrm{N}$ & $\mathrm{N}$ & $\mathrm{N}$ & $\mathrm{N}$ & $\mathrm{N}$ & $\mathrm{N}$ \\
\hline
\end{tabular}

\subsection{Establishment of baseline}

128 negative samples were collected to establish baseline. Every extend primer was along with 250 copies competitive sequences which were synthesized to evaluate the expression of fusion gene. After calculating the median and variance of 128 samples, $Z$ scores of each fusion type were deduced by following formula and the median and variance of $A L K$, ROS1 and RET are listed in Table 2 .

Table 4 Summary of fusion detection results in clinical samples. $N=$ negative

\begin{tabular}{|c|c|c|c|}
\hline 01 & ALK & EML4-ALK(E13, A20) & EML4-ALK (E13, A20) \\
\hline 02 & ALK & EML4-ALK (E13, A20) & EML4-ALK (E13, A20) \\
\hline \multirow[t]{3}{*}{03} & ALK\&ROS1 & EML4-ALK (E13, A20) & EML4-ALK (E18, A20) \\
\hline & & SLC34A2-ROS1(S4-R34) & SLC34A2-ROS1(S4-R32) \\
\hline & & SLC34A2-ROS1(S4-R32) & \\
\hline 05 & ALK & EML4-ALK (E13, A20) & EML4-ALK (E13, A20) \\
\hline 06 & ALK & EML4-ALK (E13, A20) & EML4-ALK (E13, A20) \\
\hline 07 & ALK & EML4-ALK (E13, A20) & EML4-ALK (E13, A20) \\
\hline \multirow[t]{2}{*}{08} & ROS1 & SLC34A2-ROS1(S4-R34) & SLC34A2-ROS1(S4-R34) \\
\hline & & SLC34A2-ROS1(S4-R32) & SLC34A2-ROS1(S4-R32) \\
\hline 09 & ALK & EML4-ALK (E13, A20) & $\mathrm{N}$ \\
\hline \multirow[t]{2}{*}{14} & ALK\&ROS1 & EML4-ALK (E13, A20) & EML4-ALK (E6, A20) \\
\hline & & SLC34A2-ROS1(S4-R34) & \\
\hline 15 & ALK & EML4-ALK (E13, A20) & $\mathrm{N}$ \\
\hline \multirow[t]{2}{*}{16} & ALK\&ROS1 & EML4-ALK (E13, A20) & $\mathrm{N}$ \\
\hline & & SLC34A2-ROS1(S4-R34) & \\
\hline 17 & $\mathrm{~N}$ & $\mathrm{~N}$ & $\mathrm{~N}$ \\
\hline 18 & $\mathrm{~N}$ & $\mathrm{~N}$ & $\mathrm{~N}$ \\
\hline 19 & $\mathrm{~N}$ & $\mathrm{~N}$ & $\mathrm{~N}$ \\
\hline 20 & $\mathrm{~N}$ & $\mathrm{~N}$ & $\mathrm{~N}$ \\
\hline 21 & $\mathrm{~N}$ & $\mathrm{~N}$ & $\mathrm{~N}$ \\
\hline 22 & $\mathrm{~N}$ & $\mathrm{~N}$ & $\mathrm{~N}$ \\
\hline 23 & $\mathrm{~N}$ & $\mathrm{~N}$ & $\mathrm{~N}$ \\
\hline 32 & $\mathrm{~N}$ & $\mathrm{~N}$ & $\mathrm{~N}$ \\
\hline 33 & $\mathrm{~N}$ & $\mathrm{~N}$ & $\mathrm{~N}$ \\
\hline 34 & $\mathrm{~N}$ & $\mathrm{~N}$ & $\mathrm{~N}$ \\
\hline
\end{tabular}


ALK_01-02
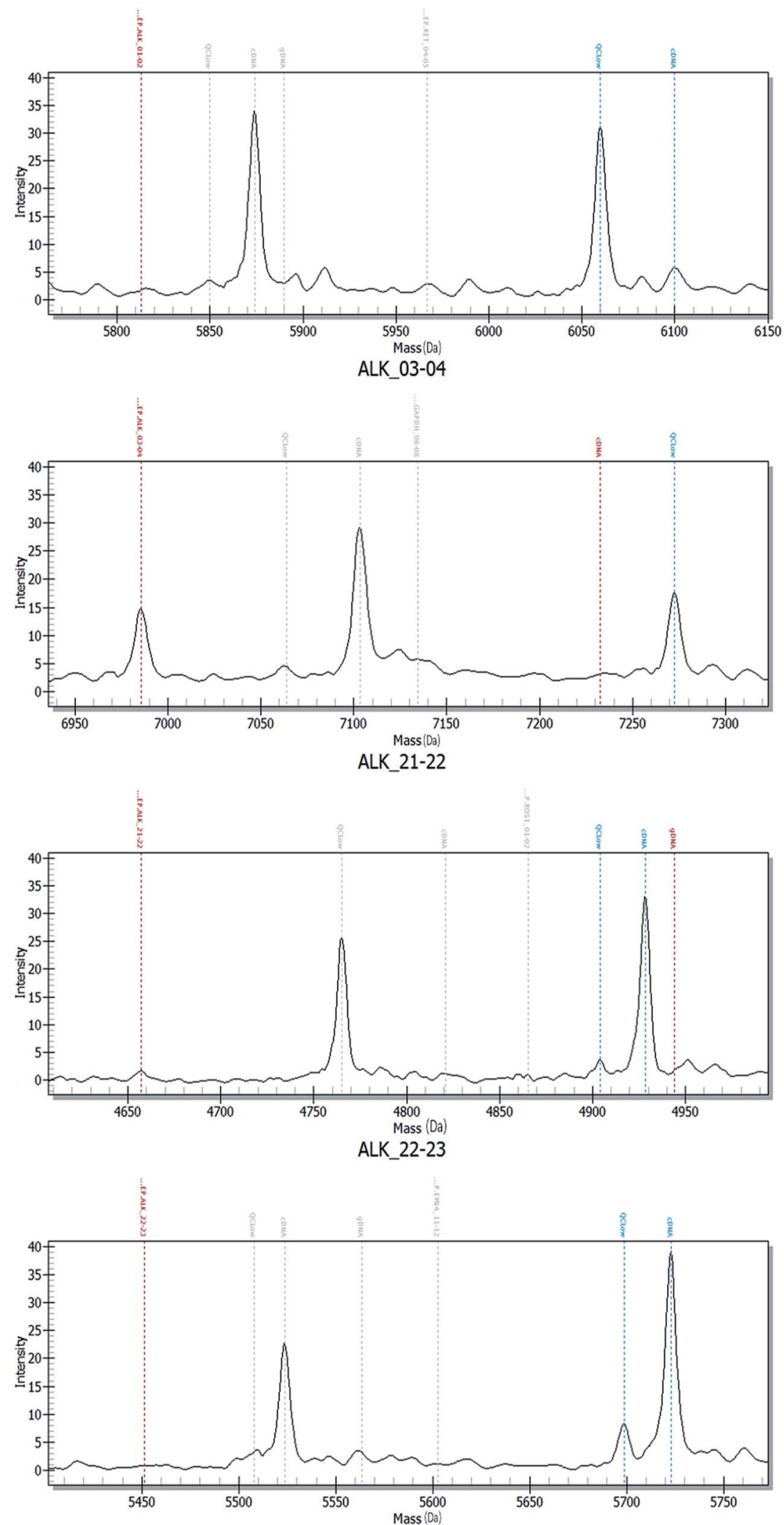

Fig. 2 Array-based MALDI-TOFMS results of ALK fusions. In the mass spectra of ALK01-02, extended primers were supposed to be detected in 6100 dalton and 7232 in ALK03-04, but no cDNA or very few cDNAs were detected in ALK 01-02 and ALK 03-04, in the mass spectra of ALK 2122, extended primers were supposed to be detected in 4929 dalton and 5722 in ALK 22-23, and large amounts of CDNA were detected in ALK 2122 and $A L K 22-23$. By calculating $Z$ score, ALK fusion was determined. 
ROS1 01-02
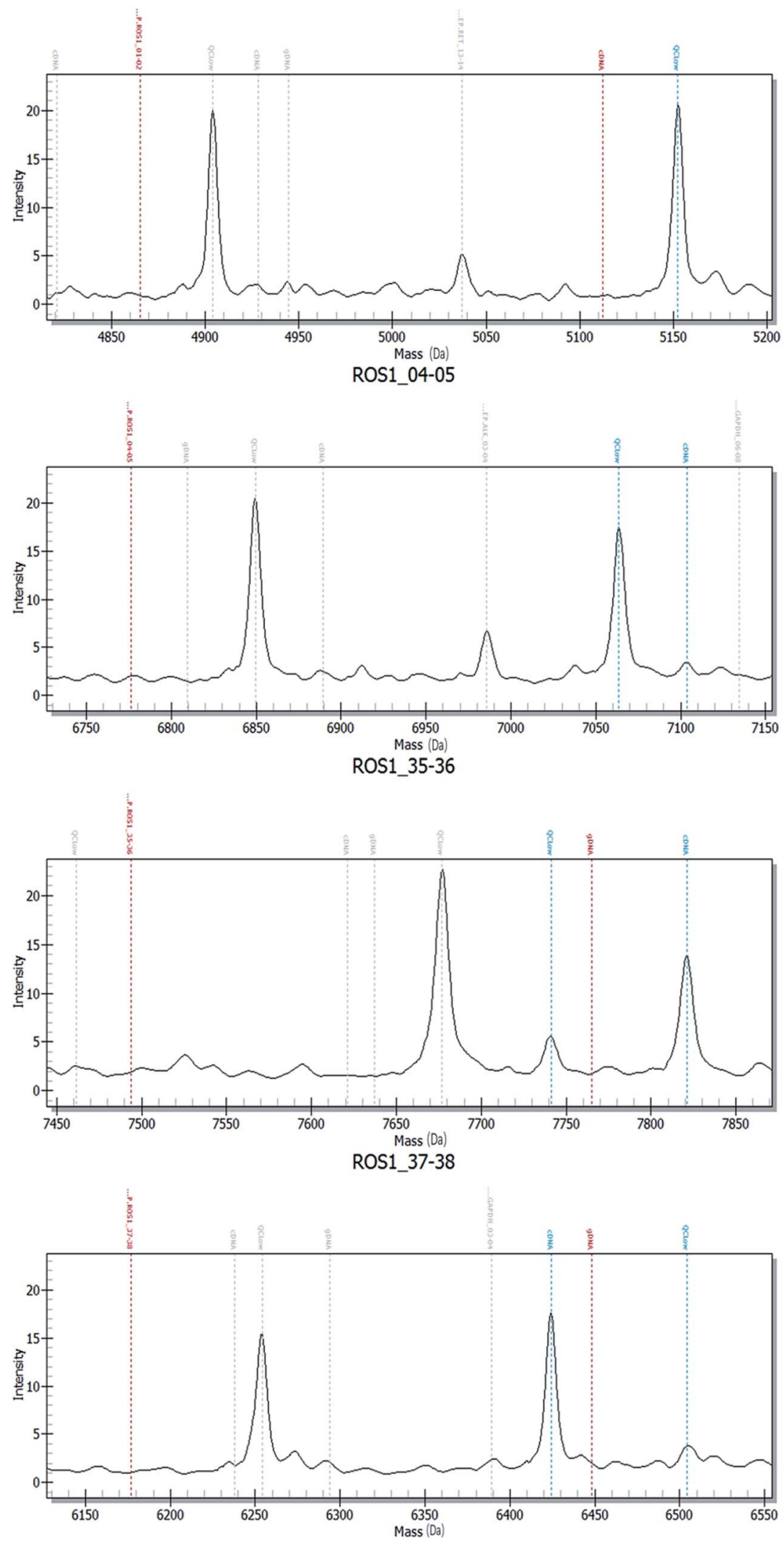

Fig. 3 Array-based MALDI-TOFMS results of ROS1 fusions. In the mass spectra of ROS1 01-02, extended primers were supposed to be detected in 5112 dalton and 7103 in ROS1 04-05, but no cDNA or very few cDNAs were detected in ROS1 01-02 and ROS1 04-05, in the mass spectra of ROS1 35-36, extended primers were supposed to be detected in 7821 dalton and 6423 in ROS1 37-38, and large amounts of cDNA were detected in ROS1 35-36 and ROS1 37-38. By calculating Z score, ROS1 fusion was determined. 
RET_02-03
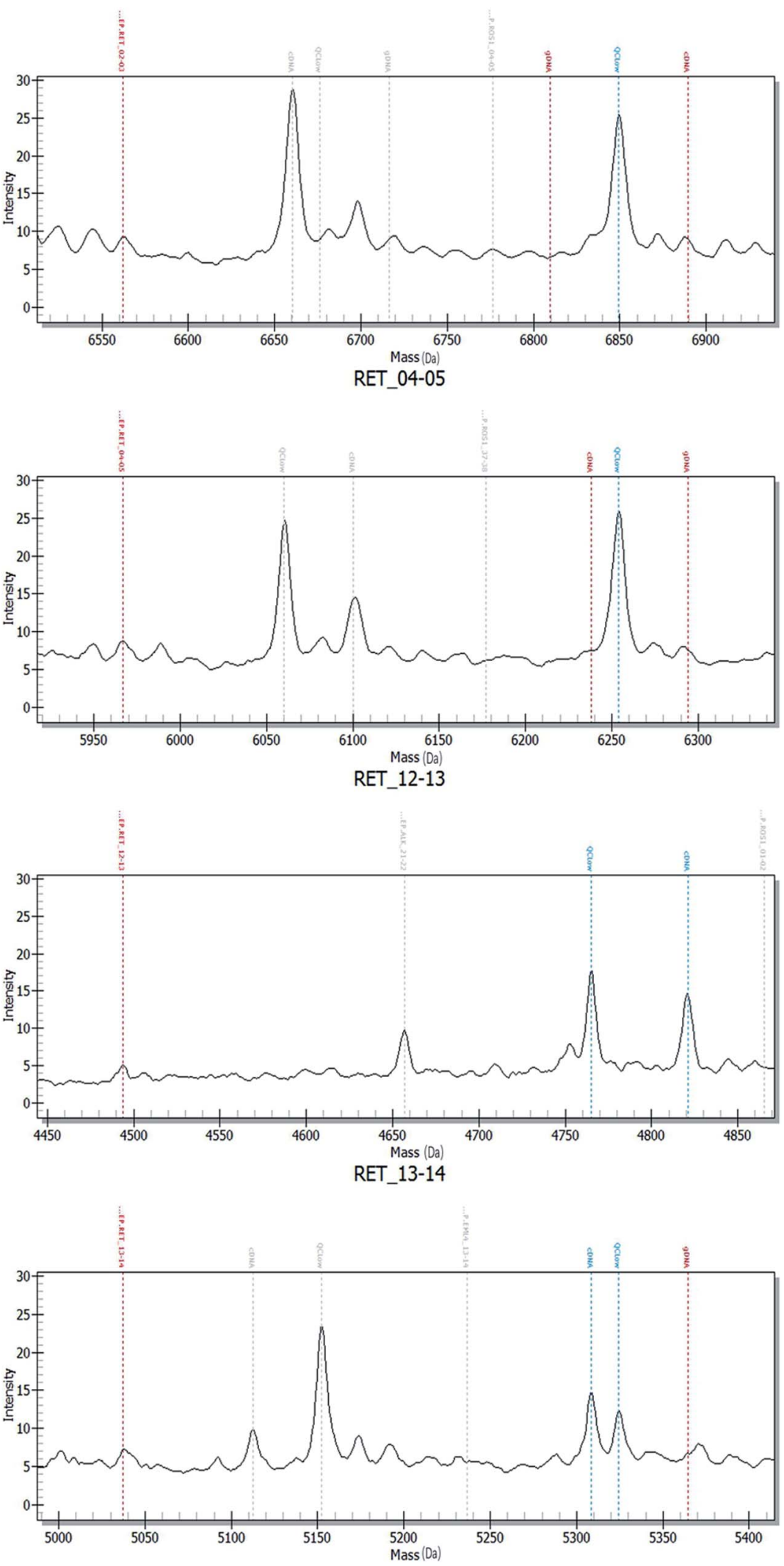

Fig. 4 Array-based MALDI-TOFMS results of RET fusions in the mass spectra of RET 02-03, extended primers were supposed to be detected in 6890 dalton and 6238 in RET04-05, but no cDNA or very few cDNAs were detected in RET 02-03 and RET 04-05, in the mass spectra of RET1213, extended primers were supposed to be detected in 4821 dalton and 5309 in RET 13-14, and large amounts of cDNAs were detected in RET 1213 and RET 13-14. By calculating $Z$ score, RET fusion was determined. 

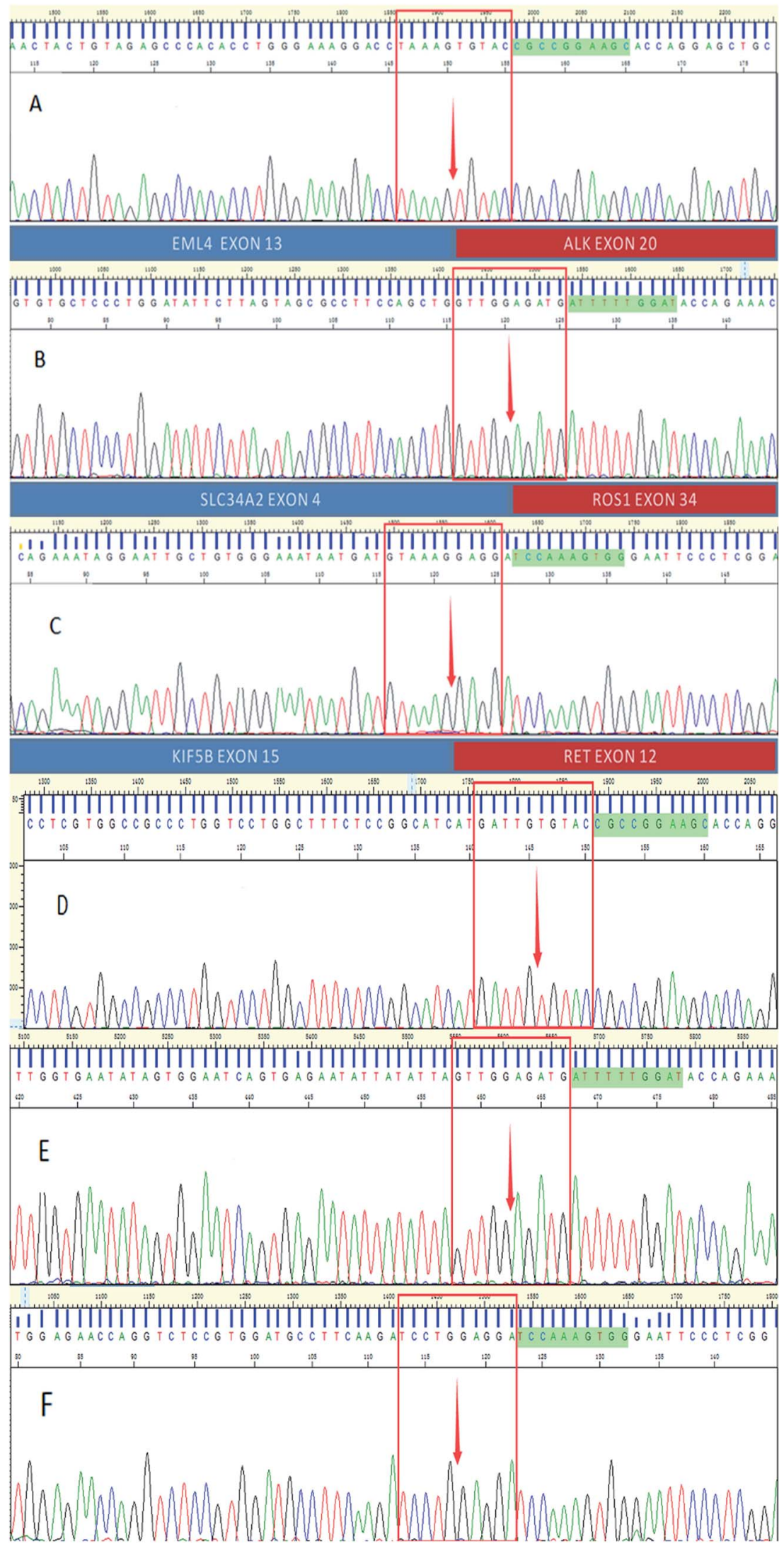

Fig. 5 Results of Sanger sequencing. A is $A L K$ fusion positive (E13, A34), the sequence before the arrow belongs to EML4, the sequence after the arrow belongs to ALK, B is ROS1 fusion positive (S4, R34), the sequence before the arrow belongs to EML4, the sequence after the arrow belongs to ROS1, C is RET fusion positive (K15, R12), the sequence before the arrow belongs to EML4, the sequence after the arrow belongs to RET, D is $A L K$ fusion negative, $\mathrm{E}$ is ROS1 fusion negative, $\mathrm{F}$ is $R E T$ fusion negative. 


$$
Z=\frac{3^{\prime} \%-\text { median }}{\mathrm{st}}
$$

when $Z$ score $\geq 3$, sample was determined as fusion positive.

\subsection{Validation of clinical samples}

A total of 34 clinical samples were tested by array-based MALDITOFMS, Sanger sequencing and NGS and the results were compared.

\section{Results}

\subsection{Results of three cell lines and negative samples}

Three cell lines (H2228, ALK positive; HCC78, ROS1 positive; LC2/AD, RET positive) and negative samples were used as positive and negative standard to establish DR MassARRAY and all these samples were also tested by Sanger sequencing and NGS. The results are listed in Table 3 .

As showed in Table 3, the results of array-based MALDI are totally in accordance with Sanger sequencing and NGS.

\subsection{Results of 34 clinical samples}

After the establishment of array-based MALDI-TOFMS, a total of 34 samples were performed fusion types detecting with three methods and the results are listed in Table 4.

As shown in Table 4, a total of 34 samples were analyzed by array-based MALDI-TOFMS, Sanger sequencing and NGS. Of these, 12 cases contain fusions gene. 7 samples were $A L K$ positive, 1 sample was $R O S 1$ positive, 4 samples had both $A L K$ and ROS1 fusions gene and no RET fusions gene were detected. Comparison of the three methods showed that the results of array-based MALDI-TOFMS were fully concordant with Sanger sequencing and $82.3 \%$ with NGS.

3.2.1 Array-based MALDI-TOFMS. Array-based MALDITOFMS is a promising method detecting fusion types by the expression of $3^{\prime}$ and $5^{\prime}$ of fusion genes. The positive results of ALK, ROS1 and RET fusion genes are as follows.

Fig. 2-4 show the results of $A L K, R O S 1$ and $R E T$ fusion positive tested by array-based MALDI-TOFMS. We tested 34 clinical samples of which 12 samples were positive for fusions. 7 were $A L K$ positive, 1 was $R O S 1$ positive and 4 were both $A L K$ and ROS1 positive, which agreed with the results from the Sanger sequencing, but was $82.3 \%$ concordant with NGS.

3.2.2 Sanger sequencing. Sanger sequencing is the gold standard for sequencing and it was performed as the standard of these samples, the results are as follows.

Fig. 5 shows the results of $A L K, R O S 1$ and $R E T$ fusion positive and negative tested by Sanger sequencing. A total of 34 samples were tested and gave the same results as array-based MALDITOFMS. Sanger sequencing provided more information than array-based MALDI-TOFMS, showing that among the $7 A L K$ fusion samples, six ALK fusions were an EML4-ALK (E13, A20) and 1 sample was an EML4-ALK (E13, A20) (E20, A20), and sample 8 was ROS1 (S4, R32) (S4, R34) fusion. 4 samples were both $A L K$ and ROS1 fusions, no RET fusion was detected.
3.2.3 Next-generation sequencing. A total of six samples gave different results between Sanger sequencing and arraybased MALDI-TOFMS. This included the exon difference in sample 03 , a false $A L K$ positive in sample 10 , a false $A L K$ negative in samples 09, 15 and 16, and a false ROS1 negative in sample 14. In conclusion, the NGS method matched $82.3 \%$ with Sanger sequencing.

\section{Discussion}

$A L K, R O S 1$ and $R E T$ fusions are important biomarkers for the detection of fusions in cancer, ${ }^{22-24}$ and a cost-effective, rapid and sensitive method that are capable of screening multiple fusions is required.

In the present study, we established a method to detect $A L K$, ROS1 and RET fusions gene in one single tube, three cell lines (H2228, ALK positive; HCC78, ROS1 positive; LC-2/AD, RET positive) and negative samples were used as standard. At the meantime, 128 negative samples were collected to establish baseline by giving median and variance, $Z$ score would be calculated to determine fusion types. Then we collected $34 \mathrm{FFPE}$ samples which were not randomly collected but partly validated by FISH previously to test this method and compared results with Sanger sequencing and NGS.

The blinded evaluation of these three methods revealed that array-based MALDI-TOFMS was fully concordant with Sanger sequencing but $82.3 \%$ concordant with NGS. However, with the limited cohort size, our statistical power for the analysis of all the three fusions was insufficient. A further assessment of these platforms with a larger number of samples is warranted.

Array-based MALDI-TOFMS requires a cDNA synthesis step, an amplification step, an annealing and single base extension step followed by MALDI-TOFMS whereby the masses of extended primers are obtained. Each extension primer was along with 250 copies competitive sequence to evaluate the $3^{\prime}$ and $5^{\prime}$ section expression of fusion gene. The $Z$ scores of each fusion would be calculated to determine fusion type of samples.

In this study, this method provides a high-throughput, sensitive and cost-effective way to detect $A L K, R O S 1$ and $R E T$ fusions. Array-based MALDI-TOFMS is an efficient method because it detects three fusion types in one tube, but Sanger sequencing needs at least 10 tubes. In addition, it requires no professional analysis which means the results are more objective. The whole process takes only one day to get results which is much faster compared with three or four days of NGS. However, this method is unable to identify specific fusion types and it is therefore limited for fundamental research. In this way, the method can not only be time and cost saving but also reduced the need of samples. However, we did not find RET fusion samples as this fusion only accounts for less than $1 \%$ and are hard to collect. ${ }^{25}$

The NGS fusion panel provides not only different fusion types but also the sequence information. Furthermore, it requires only $10 \mathrm{ng}$ of total RNA, 10 -fold less RNA than arraybased MALDI-TOFMS requires. Ali and co-authors suggested that NGS could be especially useful for detecting $A L K$ rearrangements. ${ }^{26}$ However, the disadvantages, such as expense and 
more effort for analysis it required are two major problems for large-scale application of NGS. ${ }^{27}$ Besides, because of the short sequence of fusion genes, NGS may not reach a precise result.

As the gold standard sequencing method, Sanger sequencing has been applied in many laboratories. Though this method did not approach a high precision in mutation detecting, it still cannot be replaced by new methods like NGS, especially when involves low-quality single-nucleotide variants and insertions or deletions $<10 \mathrm{bp}^{28-30}$ The reasons why Sanger sequencing has not been used in large-scale application are the high cost and professional results analysis. ${ }^{31}$

Progression-free survival was significantly longer with targeted medicine than chemotherapy. For examples, patients harboring ALK positive lung cancer treated with crizotinib got a longer progression-free survival than chemotherapy. ${ }^{32}$ And crizotinib to ROS1 fusion, ${ }^{33}$ alectinib for RET fusion also approached good effect. ${ }^{34}$ Fusion gene detecting is vital for patients to get a reasonable treatment. Besides, it can also optimize the clinical resource, reduce the pain and save cost. Methods for detecting fusion gene largely applicated in clinical are FISH and IHC, however, these two methods are limited in throughput, sensitive and cost. FISH and IHC detect one fusion of samples a time, and according to the study of Yi-cheng $\mathrm{Wu}$, these two methods are less sensitive. ${ }^{35}$ Besides, these two methods are expensive, about 7200yuan for FISH and 1950yuan for IHC. ${ }^{36}$ Array-based MALDI-TOF is quite a method to overcome the three problems. It detects three fusion gene of 96 samples a time and according to our study, it reached a high accordance with Sanger sequencing, lastly, it costs less than 100yuan per sample.

\section{Conclusion}

We established a high-throughput, sensitive and cost-effective screening method for detecting $A L K$, ROS1 and RET fusions which expended the application of mass spectra. Our findings validate this promising method that is a favorable alternative to FISH testing. Although the further evaluation with a larger number of samples would be carried. The developed method showed the potential in single-nucleotide variants, ctDNA detecting and may also be applied to the diagnosing, monitoring and prognosis of illness.

\section{Conflict of interest}

None.

\section{Acknowledgements}

The work was supported by the National Natural Science Foundation of China (grant number 21575058, 30901382), 2014 Guangzhou Healthy Care Synergy Innovate Major Special Project (2014) and 2014 Guangzhou Technology Project Cooperation Foundation (2014). The Special Project of International Scientific and Technological Cooperation in Guangzhou Development District (2017GH01).

\section{References}

1 C. S. Dela Cruz, L. T. Tanoue and R. A. Matthay, Lung Cancer: Epidemiology, Etiology, and Prevention, Clin. Chest Med., 2011, 32(4), 605-644.

2 R. Siegel, D. Naishadham and A. Jemal, Cancer statistics, 2013, Ca-Cancer J. Clin., 2013, 63(1), 11-30.

3 Y. Chen, S. Han, M. Zheng, Y. Xue and W. Liu, Clinical Characteristics of 274 Non-Small Cell Lung Cancer Patients in China, Onkologie, 2013, 36(5), 3.

$4 \mathrm{H}$. Wakelee, K. Kelly, M. J. Edelman, 50 Years of Progress in the Systemic Therapy of Non-Small Cell Lung Cancer, American Society of Clinical Oncology Educational Book, 2014, vol. 34, pp. 177-189.

5 E. L. Kwak, Y. Bang, D. R. Camidge, A. T. Shaw, B. Solomon, R. G. Maki, et al., Anaplastic Lymphoma Kinase Inhibition in Non-Small-Cell Lung Cancer, N. Engl. J. Med., 2010, 363(18), 1693-1703.

6 D. W. Wong, E. L. Leung, K. K. So, I. Y. Tam, A. D. Sihoe, L. Cheng, et al., TheEML4-ALK fusion gene is involved in various histologic types of lung cancers from nonsmokers with wild-typeEGFR andKRAS, Cancer-Am Cancer Soc, 2009, 115(8), 1723-1733.

7 D. R. Camidge, Y. Bang, E. L. Kwak, A. J. Iafrate, M. VarellaGarcia, S. B. Fox, et al., Activity and safety of crizotinib in patients with ALK-positive non-small-cell lung cancer: updated results from a phase 1 study, Lancet Oncol., 2012, 13(10), 1011-1019.

8 K. Bergethon, A. T. Shaw, S. Ou, R. Katayama and C. M. Lovly, ROS1 Rearrangements Define a Unique Molecular Class of Lung Cancers, J. Clin. Oncol., 2012, 30(8), 863-870.

9 A. T. Shaw, S. I. Ou, Y. Bang, D. R. Camidge, B. J. Solomon, R. Salgia, et al., Crizotinib inROS1 -Rearranged Non-SmallCell Lung Cancer, N. Engl. J. Med., 2014, 371(21), 1963-1971.

10 J. S. Seo, Y. S. Ju, W. C. Lee, J. Y. Shin, J. K. Lee, T. Bleazard, et al., The transcriptional landscape and mutational profile of lung adenocarcinoma, Genome Res., 2012, 22(11), 21092119.

11 D. Lipson, M. Capelletti, R. Yelensky, G. Otto, A. Parker, M. Jarosz, et al., Identification of new ALK and RET gene fusions from colorectal and lung cancer biopsies, Nat. Med., 2012, 18(3), 382-384.

12 A. T. Shaw, D. Kim, K. Nakagawa, T. Seto, L. Crinó, M. Ahn, et al., Crizotinib versus Chemotherapy in AdvancedALK -Positive Lung Cancer, N. Engl. J. Med., 2013, 368(25), 2385-2394.

13 T. Rogers, G. M. Arnau, G. L. Ryland, S. Huang, M. E. Lira, Y. Emmanuel, et al., Multiplexed transcriptome analysis to detect ALK, ROS1 and RET rearrangements in lung cancer, Sci. Rep., 2017, 7, 42259.

14 M. Soda, S. Takada, K. Takeuchi, Y. L. Choi and M. Enomoto, A mouse model for EML4-ALK-positive lung cancer, Proc. Natl. Acad. Sci. U. S. A., 2008, 105(50), 19893.

15 J. M. Boland, S. Erdogan, G. Vasmatzis, P. Yang, L. Tillmans, M. Erickson-Johnson, et al., Anaplastic lymphoma kinase immunoreactivity correlates with ALK gene rearrangement 
and transcriptional up-regulation in non-small cell lung carcinomas, Hum. Pathol., 2009, 40(8), 1152-1158.

16 A. F. Evangelista, M. F. Zanon, A. C. Carloni, F. E. de Paula, M. A. Morini, M. Ferreira-Neto, et al., Detection of ALK fusion transcripts in FFPE lung cancer samples by NanoString technology, BMC Pulm. Med., 2017, 17(1), 86.

17 S. Jadhav, M. Bhave and E. A. Palombo, Methods used for the detection and subtyping of Listeria monocytogenes, $J$. Microbiol. Methods, 2012, 88(3), 327-341.

18 D. Triest, D. Stubbe, K. De Cremer, D. Piérard, A. Normand, R. Piarroux, et al., Use of Matrix-Assisted Laser Desorption Ionization-Time of Flight Mass Spectrometry for Identification of Molds of the Fusarium Genus, J. Clin. Microbiol., 2015, 53(2), 465-476.

19 J. C. Joyner, K. D. Keuper and J. A. Cowan, Analysis of RNA cleavage by MALDI-TOF mass spectrometry, Nucleic Acids Res., 2013, 41(1), e2.

20 N. Kanaji, S. Bandoh, T. Ishii, A. Tadokoro, N. Watanabe, T. Takahama, et al., Detection of EML4-ALK fusion genes in a few cancer cells from transbronchial cytological specimens utilizing immediate cytology during bronchoscopy, Lung Cancer, 2012, 77(2), 293-298.

21 P. Wijesinghe, G. Bepler and A. Bollig-Fischer, A Mass Spectrometry Assay to Simultaneously Analyze ROS1 and RET Fusion Gene Expression in Non-Small-Cell Lung Cancer, J. Thorac. Oncol., 2015, 10(2), 381-386.

22 K. Takeuchi, Y. L. Choi, Y. Togashi, M. Soda, S. Hatano, K. Inamura, et al., KIF5B-ALK, a Novel Fusion Oncokinase Identified by an Immunohistochemistry-based Diagnostic System for ALK-positive Lung Cancer, Clin. Cancer Res., 2009, 15(9), 3143-3149.

23 K. Rikova, A. Guo, Q. Zeng, et al., Global Survey of Phosphotyrosine Signaling Identifies Oncogenic Kinases in Lung Cancer, Cell, 2007, 131(6), 1190-1203.

24 C. Tomuschat and P. Puri, RET gene is a major risk factor for Hirschsprung's disease: a meta-analysis, Pediatr. Surg. Int., 2015, 31(8), 701-710.

25 K. Takeuchi, M. Soda, Y. Togashi, R. Suzuki, S. Sakata, S. Hatano, et al., RET, ROS1 and ALK fusions in lung cancer, Nat. Med., 2012, 18(3), 378-381.

26 S. M. Ali, T. Hensing, A. B. Schrock, J. Allen, E. Sanford, K. Gowen, et al., Comprehensive Genomic Profiling Identifies a Subset of Crizotinib-ResponsiveALK
-Rearranged Non-Small Cell Lung Cancer Not Detected by Fluorescence In Situ Hybridization, Oncologist, 2016, 21(6), 762-770.

27 I. Dagogo-Jack and A. T. Shaw, Screening forALK Rearrangements in Lung Cancer: Time for a New Generation of Diagnostics?, Oncologist, 2016, 21(6), 662-663.

28 E. Vuttariello, M. Borra, C. Calise, E. Mauriello, S. Greggi, A. Vecchione, et al., A New Rapid Methodological Strategy to Assess BRCA Mutational Status, Mol. Biotechnol., 2013, 54(3), 954-960.

29 S. P. Strom, H. Lee, K. Das, E. Vilain, S. F. Nelson, W. W. Grody, et al., Assessing the necessity of confirmatory testing for exome-sequencing results in a clinical molecular diagnostic laboratory, Genet. Med., 2014, 16(7), 510-515.

30 D. Dacheva, R. Dodova, I. Popov, T. Goranova, A. Mitkova, V. Mitev, et al., Validation of an NGS approach for diagnostic BRCA1/BRCA2 mutation testing, Mol. Diagn. Ther., 2015, 19(2), 119-130.

31 S. Lee, S. Zhou, T. Zhou and G. Hong, Sanger Sequencing for BRCA1 c.68_69del, BRCA1 c.5266dup and BRCA2 c.5946del Mutation Screen on Pap Smear Cytology Samples, Int. J. Mol. Sci., 2016, 17(2), 229.

32 B. J. Solomon, T. Mok, D. Kim, Y. Wu, K. Nakagawa, T. Mekhail, et al., First-Line Crizotinib versus Chemotherapy inALK-Positive Lung Cancer, N. Engl. J. Med., 2014, 371(23), 2167-2177.

33 A. T. Shaw, S. I. Ou, Y. Bang, D. R. Camidge, B. J. Solomon, R. Salgia, et al., Crizotinib inROS1-Rearranged Non-SmallCell Lung Cancer, N. Engl. J. Med., 2014, 371(21), 1963-1971.

34 T. Kodama, T. Tsukaguchi, Y. Satoh, M. Yoshida, Y. Watanabe, O. Kondoh, et al., Alectinib Shows Potent Antitumor Activity against RET-Rearranged Non-Small Cell Lung Cancer, Mol. Cancer Ther., 2014, 13(12), 2910-2918.

35 Y. Wu, Comparison of IHC, FISH and RT-PCR Methods for Detection of ALK Rearrangements in 312 Non-Small Cell Lung Cancer Patients in Taiwan, PLoS One, 2013, 8(8), e70839.

36 H. Jiang-jiang, L. Xian-zhong and H. Da, Economic Analysis on Anaplastic Lymphoma Kinase Related Companion Diagnosis Methods before the Targeted Therapy for Nonsmall Cell Lung Cancer, Chinese Health Economics, 2017, 36(1), 40-43. 